\title{
A Study on the Evolution of Famous Car Symbols in the 20th Century
}

\author{
Zongyuan Bian ${ }^{1, \mathrm{a}}$ and Yang $\mathrm{Du}^{2, \mathrm{~b}}$ \\ ${ }^{1,2}$ School of Architecture and Art Design, Beijing Jiaotong University, Beijing 100044, China \\ a807249775@qq.com, b799690386@qq.com
}

\begin{abstract}
Based on the case of 30 famous automobile symbols in the world, an image segmentation method based on car symbol is proposed in this paper, and the evolution of symbolic elements in the twentieth century are made a more accurate classification and identification. The trend study is simultaneous with the evolution study. Through a large number of numerical summary analyses, the evolution characteristic elements and similarities of the samples in the same age and the sample evolution type of different ages are divided, a more accurate law of evolution of symbol is obtained by using semantic differences analysis point of view, which is helpful for the later symbol analysis and design.
\end{abstract}

Keywords: Automobile symbol; Segmentation method; Evolutionary feature; Symbolic trend

\section{二十世纪著名汽车标志符号演变研究}

\author{
边宗圆 ${ }^{1}$ 杜洋 $^{2}$
}

(1.2. 北京交通大学建筑与艺术学院, 北京市, 100044)

摘要: 以三十个世界上著名汽车标志的案例为样本, 提出一种基于汽车标志的图像切分研究法, 对二十世纪汽车标志的 符号要素演变情况进行比较准确的分类比较和识别, 进行演变研究的同时进行趋势研究。通过大量的数字总结分析, 划分出 演变特征要素和相同年代样本之间相通点以及不同年代样本演变类型, 使用语义学差异性分析法观点得出一个比较准确的标 志符号演变规律，从而对后期标志分析和设计起到帮助的作用。

关键词: 汽车标志; 切分法; 演变特征; 符号趋势

中图分类号: J524.4 文献标志码: A

引言

当今各大汽车公司都已开始从品牌战略的高度来规划和使用汽车标志, 都在不断改进汽车标志设计, 以达到传承、巩固、改善和传播品牌形象的目的 ${ }^{[1]}$ 。汽车标志的造型要素主要依赖符号的构成形式, 英文 文字以及色彩搭配组成, 这里的研究暂且不谈企业文化背景的影响, 仅从标志符号本身出发, 研究拟通过 不同品牌, 不同国家的汽车标志符号构成要素的变化对比研究, 结合切分法研究方式, 探究出这些形式要 素之间的相互关系以及要素变化产生的效果和时代的关系以及演变规律, 并合理的预测标志的演变趋势。

\section{1 研究样本采集}

通过网上搜集资料，收集了二十世纪期间一些著名汽车品牌更换标志的时间和改变前后图片，依据汽 车企业标志改变前后的变化形式比较有代表性特征和在行业中的比较具有影响力和权威性, 最终确定了奔 驰, 宝马等 30 家著名汽车企业标志构成要素变更对比图作为研究样本, (图一) 研究重点关注的是 “汽车 标志要素在改变中的变化之处” 重点, 而不是 “过去和现在的标志是什么样子” 保证较正确的反映出演变 
规律和趋势。(图二)

\section{2 汽车标志构成要素的演变分析}

\section{1 演变类型和要素设定}

通过与已具备多年标志设计工作经验和汽车品牌研究的 5 名业界知名人士探讨, 他们通过集体讨论法 和试错法 ${ }^{[2]}$ 以及形态分析法 ${ }^{[3]}$ 将一百年间的 30 个品牌数据进行对比分析, 演变分析。探讨标志符号的结构, 颜色, 字体的演变特征, 在关于变更条件的设定展开了激烈
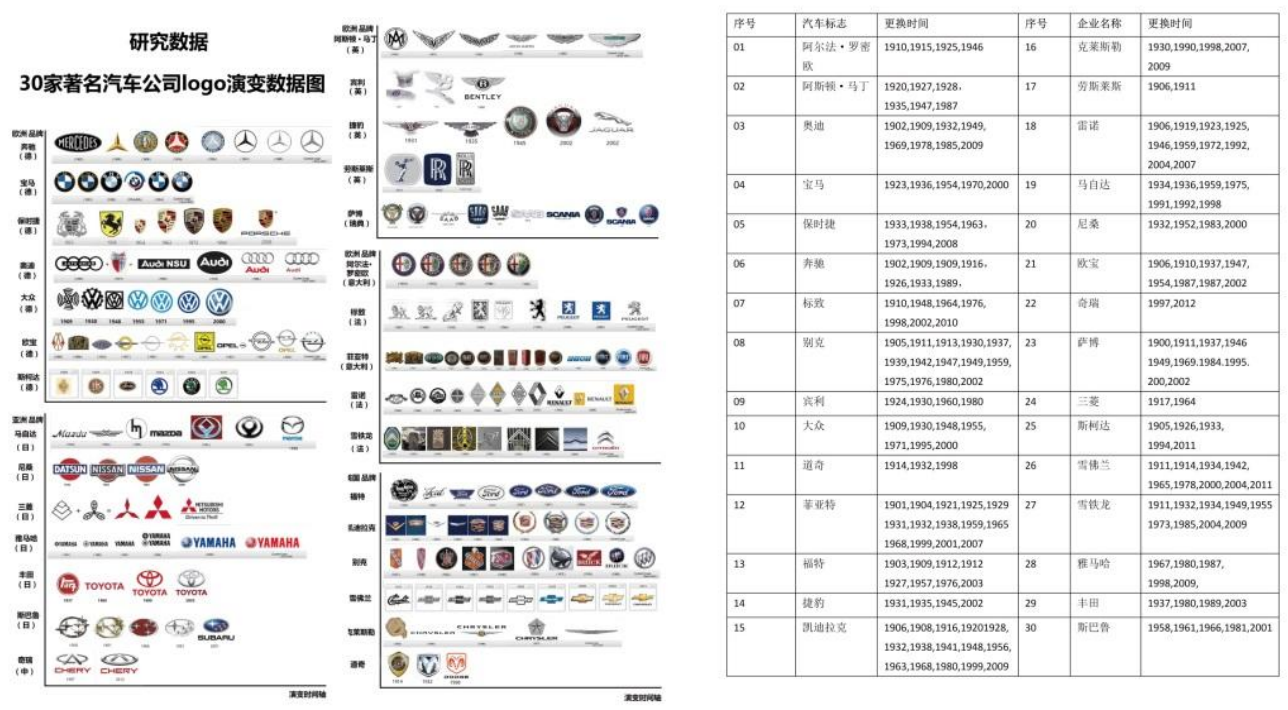

图一 三十家著名汽车企业更换标志的样本 图二汽车企业更换标志的时间样本

的讨论, 若只是研究每个时期的汽车标志特征, 那么演变变化难以总结规律, 最后保证在各个要素单独保 证合理准确且独特性的原则下, 分析汽车更改标志的符号之间的相关性和改变的要素。

分析包含几项基本原则: (1) 重点关注演变趋势, 即主要演变要素, 未变化或变化不能影响主体要素 的特征这里不作考虑。(2) 标志变化只研究视觉符号的变化, 即对于汽车品牌文化, 企业内部文化以及社 会经济文化要素不做考虑。(3) 变化要素互不干扰, 即研究某要素就是唯一的, 不能造成标志图形的一个 要素演变关联了数据的多个要素点, 这样才能将切分的效果表现出来, 确保结果的准确性。(4) 演变的特 征设计要具体贴近汽车标志, 例如汽车标志的外形轮廓较多的是圆形和盾形, 而不会存在菱形或三角形, 这样能使研究更加具体。

最后归纳总结出在色彩, 图形, 文字三个维度的十一种不同要素的变更要素图 (图三)

\begin{tabular}{|c|c|c|c|c|c|}
\hline & & 1 & 2 & 3 & 4 \\
\hline \multirow{4}{*}{ A } & 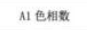 & 无筫化 & 更丰高 & 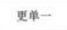 & \\
\hline & A2色相 & 无変化 & 政蕰 & 变黄弶 & 变果白 \\
\hline & A3 触 & 无变化 & 变明竞 & 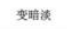 & \\
\hline & M蛙度 & 无变化 & 更明护 & 更䜿重 & \\
\hline \multirow{4}{*}{ B 图形 } & B1 要素份䚁量 & 无变化 & 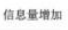 & 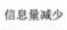 & \\
\hline & B2 湈挍 & 无变化 & 变丰言 & 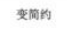 & 效 \\
\hline & 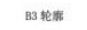 & 无采化 & 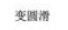 & 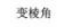 & 效 \\
\hline & 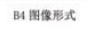 & 无带化 & 主整复化 & 变霍化 & 形式受化 \\
\hline \multirow{3}{*}{ c 文傮 } & c1 文辛字体 & 无变化 & 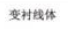 & 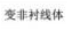 & 效 \\
\hline & 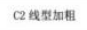 & 无复化 & 加相 & tetit & \\
\hline & C3 䇰休样式 & 无复化 & 委国形化 & 活政变正式 & \\
\hline
\end{tabular}




\section{1.1 色彩维度特征演变}

A1 色相数：体现在无变化, 变丰富和变单一三个变化形式; A2 色相: 从十六色环中提取样本中主要 出现的色彩, 进行统计, 大致分为红, 蓝, 黄, 绿四个区间色, 由于在汽车标志色彩搭配中蓝色和红色, 黄色和绿色搭配最为常见, 数量最多, 故变化分为红蓝色, 黄绿色; A3 明度: PCCS 将明度分为 9 阶, 为 了研究直观性, 明度就划分为更明亮或暗淡; A4 纯度: 保证演变的客观性, 将纯度演变划分为更厚重或是 更明快。

\section{1.2 图形维度特征演变}

B1 要素信息量: 样本中要素信息量包含主体图案以及辅助图形还有包含底部背景以及底纹还有轮廓这 几个方面的要素; B2 底纹: 底纹可能变复杂, 或增加数量或增加样式, 或反之; B3 轮廓: 复对比研究发 现最多的就两个趋势或变圆滑, 或变棱角, 或变无; B4 图像形式: 主题变化或形式变化。

\section{1 .3 文字维度特征演变}

C1 文字字体: 这里主要考虑祄线体和非祄线体; C2 线性粗细: 字体的粗细对于品牌识别有很重要的 影响性，能从中反应文字所占比重；C3 字体样式：字体形式变正式或变活泼或变无。

\section{2 样本演变特征归纳统计分析及趋势分析}

根据标志符号演变特征要素与变化形式表格要素将样本变化特征列入参考时限内, 每十年为一个单 位, 以品牌为系谱轴, 以图三的三个维度的十一个演变特征为毗邻轴, 建立一个参考关系演变图。(图四 数 据统计结果)

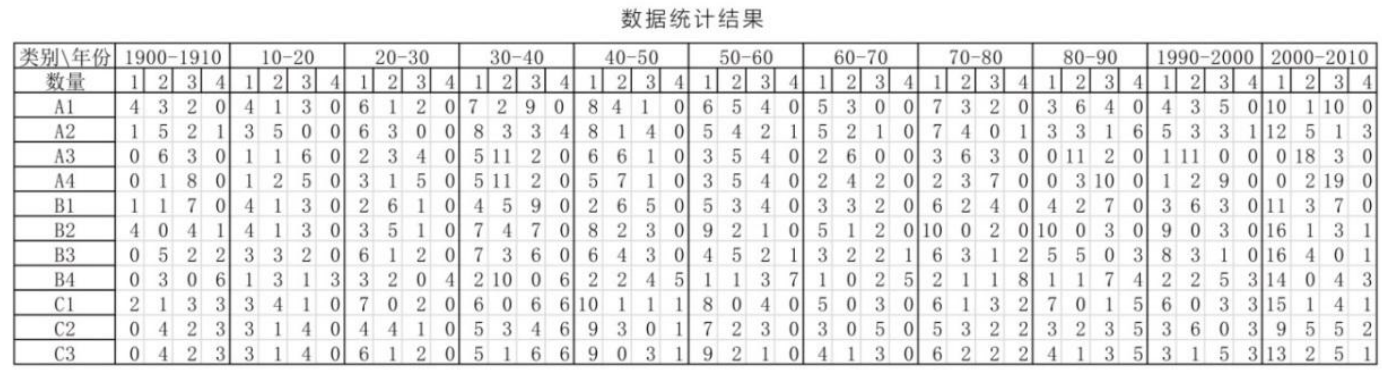

（1）色相单一化发展：从 1980 年之后到现在，车标的单一化十分明显。（2）色相趋于稳定，且一部 分趋于无色: 红蓝色从 50 年代开始变成上升并逐渐稳定, 黄绿色在 30 年代到 70 年代活跃了一段时间, 但是后期就消退了, 而无色相的趋势很明显的上升, 并成为一种主流。(3) 色彩明度呈现明亮趋势: 从 1950 年开始到现在车标变得更加明亮了, 车标应用了阴影立体化表现出质感, 高光明显, (4) 色彩纯度向厚重 感发展: 从 1900 到 1950 年色彩明度逐渐由色彩纯度厚重变向明快化, 从1950 年开始厚重的纯度开始盛 行。（5）要素信息量减少且趋于稳定：从 1900 到 1920 要素量减少, 从 1920-1950 年要素信息量又增加, 从 1960 年开始要素信息量简少的个数增多并在 21 世纪初达到稳定。(6) 底纹变简约并有消失的趋势: 在 100 年的车标设计变化当中, 车标底纹逐步演变简约。(7) 标志轮廓圆滑化发展: 在 100 年间主要趋势是 圆滑化发展, 并开始出现外部轮廓消逝的趋势, 逐步只剩下标志主体, 外部圆滑或消失。(8) 图像形式简 
约化形式发展: 在这 100 年中, 图形形式主要是发生主题变化, 后期主要发生在形式变化上。（9）车标字 体趋于非祄线体字体: 大部分时间发展都在朝着非祄线体方向发展, 并且逐渐发展为省去字体的趋势。(10) 车标字体线型朝着变细方向发展: 从 1900 到 1950 年, 车标字体线型都在变粗, 从 1950 年开始字体线型 开始变细, 但在 90 年代忽然变粗, 随后又兴起细线型。（11）文字字体趋于更加正式化：把文字直接作为 标志的现象也在消失，这就表现出来字体逐渐表现为正式化，通用化和简单化。

\section{3 总结}

汽车标志的更换是汽车企业的重大决策, 将影响着品牌影响力, 达到企业形象与传播效果的提升, 当 然车标的演变紧随生产力和社会的步伐 ${ }^{[4]}$ 。文章通过研究二十世纪 30 家著名汽车企业发布的汽车标志变化 发展对比研究, 总结出汽车标志的色相朝着单一性和灰度模式发展, 企业标志的色相发展趋于稳定, 色彩 逐渐变化为更加明亮厚重, 在汽车标志的图形上, 演变的更加简约, 形式变化更加简化, 表现直接, 底 纹简约趋于消失, 外轮廓更加圆滑, 标志的字体趋势为非祄线体和正式化, 字体线型演变的更加纤细, 同 时字体在标志中占的力量在减弱。可以看出汽车标志的发展进程是螺旋式上升, 波浪式前进的 ${ }^{[5]}$ 。通过总 结汽车标志符号的演变规律，希望对汽车标志的设计和发展提供一定的理论参考。

\section{参考文献:}

[1] 李健. 汽车标志设计研究 [D]. 吉林大学, 2012.

[2] 文援朝, 胡慧河. 波普尔试错法述评 [J]. 求索, 2002, 02:87-89.

[3] 曾清旗, 林铭泉, 萧世文, 陈龙安, 应用感性语意及意象认知于电脑辅助银行业几何形商标设计之建构 [J]设计学 报 VOL9，2005（3): 97-109.

[4] 韩寒. 从汽车标志发展看极简主义的影响 [J]. 美与时代 (中), 2012, 04:78-79.

[5] 法尔曼.色彩物语：影响力的秘密：第 2 版 [M]. 北京：人民邮电出版社，2012.1

[6] 孙虎. 导入标志元素的汽车车身造型设计研究 [J]. 装饰, 2010, 11:98-99.

[7] 杨先艺. 设计社会学 $[M]$. 北京: 中国建筑工业出版社, 2014.7

[8] 李健. 汽车标志设计研究[D]. 吉林大学, 2012.

[9] 于洪伟. 汽车标志识别的研究[D]. 沈阳工业大学, 2007.

[10］李响. 符号学在标志设计中的应用研究 [D]. 昆明理工大学, 2007.

\section{References:}

[1] Li Jian. Automobile Symbol Design Study [D]. Jilin University, 2012.

[2] Wen Yuanzhao, Hu Huihe. Popper Trial and Error Method Review [J]. Seeking, 2002, 02: 87-89.

[3] Zeng Qingqi, Lin Mingquan, Xiao Shiwen, Chen Longan, The Construction for Appling Perceptual Semantic and Imagery Cognition in Computer-aided Banking Geometric Trademark Design [J] Design Journal Vo192005 (3): 97-109.

[4] Han Han. The View of Minimalist Influence from the Development of Car Symbol [J]. Beauty and the Times (middle), 2012, 04: 78-79.

[5] Farman. Color Monogatari: The Secret of Influence: the $2^{\text {nd }}$ Edition [M]. Beijing: People's Posts and Telecommunications Press, 2012.1

[6] Sun Hu. Study on Lead-in Symbol Elements of Car Body Design [J]. Decorative, 2010,11: 98-99. 
[7] Yang Xianyi. Design Sociology [M]. Beijing: China Construction Industry Press, 2014.7

[8] Li Jian. Automobile Symbol Design Research [D]. Jilin University, 2012.

[9] Yu Hongwei. Automobile Symbol Recognition Research [D]. Shenyang University of Technology, 2007.

[10]Li Xiang. Study on the Application of Semiotics in Symbol Design [D]. Kunming University of Science and Technology, 2007. 